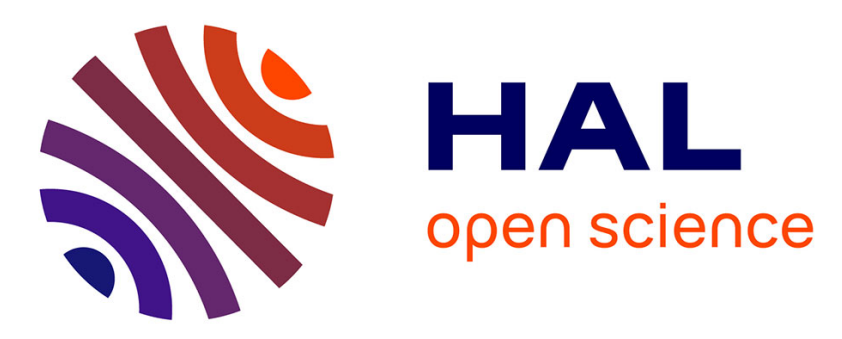

\title{
On Equations of the Linear Theory of Shells with Surface Stresses Taken into Account
}

\author{
Holm Altenbach, Victor A. Eremeyev, Nikita F. Morozov
}

\section{To cite this version:}

Holm Altenbach, Victor A. Eremeyev, Nikita F. Morozov. On Equations of the Linear Theory of Shells with Surface Stresses Taken into Account. Mechanics of Solids, 2010, 45 (3), pp.331-342. hal00824841

\section{HAL Id: hal-00824841 \\ https://hal.science/hal-00824841}

Submitted on 22 May 2013

HAL is a multi-disciplinary open access archive for the deposit and dissemination of scientific research documents, whether they are published or not. The documents may come from teaching and research institutions in France or abroad, or from public or private research centers.
L'archive ouverte pluridisciplinaire HAL, est destinée au dépôt et à la diffusion de documents scientifiques de niveau recherche, publiés ou non, émanant des établissements d'enseignement et de recherche français ou étrangers, des laboratoires publics ou privés. 


\title{
On Equations of the Linear Theory of Shells with Surface Stresses Taken into Account
}

\author{
H. Altenbach ${ }^{*}$, V. A. Eremeev ${ }^{2 * * *}$, and N. F. Morozov ${ }^{3 * * * *}$ \\ ${ }^{1}$ Martin Luther University Halle-Wittenberg, \\ Kurt-Mothes 1, Halle (Saale), 06099 Germany \\ ${ }^{2}$ South Federal University, \\ Mil'chakova 8a, Rostov-on-Don, 344090 Russia \\ ${ }^{3}$ St. Petersburg State University, \\ Universitetskaya nab.7-9, St. Petersburg, 199034 Russia
}

Received August 21, 2009

\begin{abstract}
We construct equations of equilibrium and constitutive relations of linear theory of plates and shells with transverse shear strain taken into account, which are based on reducing the spatial elasticity relations with surface stresses taken into account to two-dimensional equations given on the shell median surface. We analyze the influence of surface elasticity moduli on the effective stiffness of plates and shells.
\end{abstract}

Key words: surface stresses, shells, plates, effective stiffnesses, surface tension.

Recently the theory of elasticity with the surface stresses taken into account has been widely used in problems of nanomechanics (e.g., see review [1]). In the framework of this theory, along with the stress tensor $\boldsymbol{\sigma}$ determined in the bulk of the body $V$ and on its surface $\Omega$, the surface stresses $\boldsymbol{\tau}$ act on the body boundary or on part of the boundary. The tensor $\tau$ is a generalization of the scalar surface tension, which is well known in hydromechanics [2], to the case of deformable solids. In particular, the introduction of surface stresses permits describing the dimensional effect typical of nanomaterials [1, 3, 4]. An example of a dimensional effect is the Young modulus dependence on the nanodimensional rod diameter [5-7].

Mathematical studies of surface stresses in solids and liquids date back to the works of Laplace [8,9], Young [10], Gibbs [11], and many other researches (e.g., see reviews in [12-16]). Mechanics of deformable solids with surface stresses taken into account was developed in [13, 17-22] and several other works. From the mechanical viewpoint, the model of surface stresses proposed in [17] is equivalent to a deformable body with an elastic membrane pasted to its surface. In the framework of the model [17], the tensor $\boldsymbol{\tau}$ can be considered as the tensor of stresses acting in this membrane. The model of [17] is generalized in [22] to the case where the body surface flexural stiffness is taken into account. Essentially, the surface stress model of [22] can be reduced to the case of a shell on the body surface, which is described by Kirchhoff-Love type equations. An analysis of surface effects including the moment surface stresses was performed in [13]. In [23], it was proposed to describe the surface stresses by equations of the theory of micropolar shells.

In the literature, in problems of mechanics of objects such as nanodimensional beams, plates, and shells, continuum approaches based on the Timoshenko, Kirchhoff-Love, Reissner-Mindlin, von Kármán models are widely used (e.g., see 24-27]). An essential element of two-dimensional theories of plates and shells is given by the modified equations of state in which the typical characteristics of the nanomaterial behavior are taken into account. Different problems of mechanics of nanodimensional plates with the surface stresses taken into account were discussed in [24, 26, 28-32].

In the present paper, which continues [32], in the framework of the linear theory of shell elasticity with the transverse shear taken into account, we obtain equations of equilibrium and constitutive relations

\footnotetext{
"e-mail: holm.altenbach@iw.uni-halle.de

**-mail: eremeyev.victor@gmail.com

e-mail: morozov@nm1016.spb.edu
} 
for stress and moment tensors with the surface stresses acting on the shell surface taken into account. The difference from the classical case, i.e., the theory without surface stresses, is that the shell effective stiffness, in particular, the flexural stiffness $D$, also depends on the surface moduli of elasticity. This results in a more complicated dependence of the shell stiffness on the thickness, which manifests itself in the case of nanodimensional thicknesses of the shell.

We use an example of a plate to discuss the similarity and distinctions of the obtained equations of state in the case of three-layer plates considered in [33-36].

\section{BASIC EQUATIONS OF LINEAR ELASTICITY WITH SURFACE STRESSES TAKEN INTO ACCOUNT}

To pose the boundary-value problems of elasticity with surface stresses, we use the variational method. We assume that the solid occupies a domain $V \in \mathbb{R}^{3}$ bounded by a smooth surface $\Omega$. Without loss of generality, we assume that part of the boundary $\Omega_{1} \subset \Omega$ is fixed, i.e., the field of the displacement vector $\mathbf{u}$ on it is zero:

$$
\left.\mathbf{u}\right|_{\Omega_{1}}=\mathbf{0} \text {. }
$$

We also assume that the external loads $\Omega_{2}=\Omega \backslash \Omega_{1}$ are given on the remaining part of the boundary $\varphi$. The action of surface stresses $\boldsymbol{\tau}$ on $\Omega_{2}$ is also taken into account.

Further, for simplicity, we consider only isotropic bodies. The potential energy of a deformed elastic body is given by the formula $W=W(\varepsilon) \equiv \frac{1}{2} \lambda \operatorname{tr}^{2} \varepsilon+\mu \varepsilon \cdot \cdot \varepsilon$, where $\varepsilon$ is the stress tensor, $\varepsilon=\varepsilon(\mathbf{u}) \equiv \frac{1}{2}\left(\nabla \mathbf{u}+\nabla \mathbf{u}^{T}\right), \lambda$ and $\mu$ are Lamé constants, $\nabla$ is the spatial gradient operator, the dot denotes the scalar product, $\varepsilon \cdot \cdot \varepsilon=\operatorname{tr}(\varepsilon \cdot \varepsilon)$, and tr is the trace operator of second-rank tensor.

In addition to $W$, we also specify the density of the surface energy $U$ as a surface strain function defined on $\Omega_{2}$. For an isotropic body, the surface energy $U$ can be written as [17]:

$$
U=U(\boldsymbol{\epsilon}) \equiv \tau_{0} \operatorname{tr} \boldsymbol{\epsilon}+\frac{1}{2} \lambda_{S} \operatorname{tr}^{2} \boldsymbol{\epsilon}+\mu_{S} \boldsymbol{\epsilon} \cdot \boldsymbol{\epsilon} .
$$

Here $\boldsymbol{\epsilon}$ is the surface strain tensor, $\boldsymbol{\epsilon}=\boldsymbol{\epsilon}(\mathbf{u}) \equiv \frac{1}{2}\left[\left(\nabla_{S} \mathbf{u}_{S}\right) \cdot \mathbf{A}+\mathbf{A} \cdot\left(\nabla_{S} \mathbf{u}_{S}\right)^{T}\right], \mathbf{u}_{S}=\left.\mathbf{u}\right|_{\Omega_{2}}$ is the displacement vector on $\Omega_{2}, \nabla_{S}$ is the surface gradient operator [23, 37], $\mathbf{A}=\mathbf{I}-\mathbf{n} \otimes \mathbf{n}, \mathbf{I}$ is the threedimensional unit tensor, $\mathbf{n}$ is the vector of outer normal on $\Omega_{2}, \lambda_{S}$ and $\mu_{S}$ are the surface elastic moduli (analogs of surface Lamé constants), and $\tau_{0}$ is the residual surface stress. The function $U$ in (1.2) is in fact the membrane shell strain energy and $\epsilon$ is the linear tensor in the tangent plane, which is used in the linear theory of shells [38-41].

The total energy functional for a body with surface stresses has the form

$$
\begin{aligned}
& J(\mathbf{u})=E(\mathbf{u})-A(\mathbf{u}), \\
& E(\mathbf{u})=\int_{V} W(\boldsymbol{\varepsilon}) d V+\int_{\Omega_{2}} U(\boldsymbol{\epsilon}) d \Omega, \quad A(\mathbf{u})=\int_{V} \mathbf{f} \cdot \mathbf{u} d V+\int_{\Omega_{2}} \boldsymbol{\varphi} \cdot \mathbf{u} d \Omega .
\end{aligned}
$$

Here $\mathbf{f}$ and $\boldsymbol{\varphi}$ are vectors of external bulk and surface forces.

The stationarity condition

$$
\delta J(\mathbf{u})=0 \quad \forall \delta \mathbf{u}:\left.\delta \mathbf{u}\right|_{\Omega_{1}}=\mathbf{0}
$$

implies the equations of equilibrium and the static boundary conditions

$$
\begin{aligned}
& \nabla \cdot \boldsymbol{\sigma}+\mathbf{f}=\mathbf{0}, \\
& \left.\left(\mathbf{n} \cdot \boldsymbol{\sigma}-\nabla_{S} \cdot \boldsymbol{\tau}\right)\right|_{\Omega_{2}}=\boldsymbol{\varphi} .
\end{aligned}
$$

The stress and surface stress tensors in (1.3) and (1.4) are given by the formulas

$$
\boldsymbol{\sigma}=\frac{\partial W}{\partial \boldsymbol{\varepsilon}}=\lambda \mathbf{I} \operatorname{tr} \boldsymbol{\varepsilon}+2 \mu \boldsymbol{\varepsilon}, \quad \boldsymbol{\tau}=\frac{\partial U}{\partial \boldsymbol{\epsilon}}=\tau_{0} \mathbf{A}+\lambda_{S} \mathbf{A} \operatorname{tr} \boldsymbol{\epsilon}+2 \mu_{S} \boldsymbol{\epsilon} .
$$

A relation of the form (1.4) was used, for example, in [1,3] for $\tau_{0}=0$.

The converse statement also holds: any solution of the boundary-value problem (1.3), (1.1), and (1.4) is a stationary point of the functional $J(\mathbf{u})$ on kinematically admissible displacement fields $\mathbf{u}$. 
coordinates on $\omega$. The radius vectors of $\Omega_{ \pm}$are, respectively, equal to $\mathbf{r}_{ \pm}=\boldsymbol{\rho} \pm \frac{1}{2} \mathbf{n} h$. In what follows, for simplicity, we assume that $h$ is constant, although the final results also remain true for variable shell thickness if $g$ is a sufficiently smooth function of the coordinates $q^{1}$ and $q^{2}$.

We write several auxiliary formulas relevant for describing tensor fields near the surface $\omega$ $[23,35,37,41]$. The main and dual bases on $\omega$ are given by the formulas $\boldsymbol{\rho}_{\alpha}=\partial \boldsymbol{\rho} / \partial q^{\alpha}, \boldsymbol{\rho}_{\alpha} \cdot \boldsymbol{\rho}^{\beta}=\delta_{\alpha}^{\beta}$, $\alpha, \beta=1,2$, where $\delta_{\alpha}^{\beta}$ is the Kronecker symbol. The surface nabla operator on $\omega$ is

$$
\nabla_{S}=\boldsymbol{\rho}^{\alpha} \frac{\partial}{\partial q^{\alpha}}
$$

As the curvilinear coordinates near $\omega$, we use the variables $q^{1}, q^{2}, z$. Then the vectors of the main and dual spatial bases in $V$ are expressed as

$$
\begin{aligned}
& \mathbf{r}_{\alpha}=\frac{\partial \mathbf{r}}{\partial q^{\alpha}}=\boldsymbol{\rho}_{\alpha}+z \frac{\partial \mathbf{n}}{\partial q^{\alpha}}=(\mathbf{A}-z \mathbf{B}) \cdot \boldsymbol{\rho}_{\alpha}, \quad \mathbf{r}_{3}=\mathbf{r}^{3}=\mathbf{n}, \\
& \mathbf{r}^{\alpha}=(\mathbf{A}-z \mathbf{B})^{-1} \cdot \boldsymbol{\rho}^{\alpha}, \quad \mathbf{r}_{\alpha} \cdot \mathbf{r}^{\beta}=\delta_{\alpha}^{\beta}, \quad \mathbf{B}=-\nabla_{S} \mathbf{n},
\end{aligned}
$$

where $\mathbf{B}$ is the tensor of curvature of the surface $\omega$. The spatial gradient operator $\nabla$ and the surface gradient operators $\nabla_{S}^{ \pm}$are, respectively, given by

$$
\begin{aligned}
& \nabla=\mathbf{r}^{\alpha} \frac{\partial}{\partial q^{\alpha}}+\mathbf{n} \frac{\partial}{\partial z}=(\mathbf{A}-z \mathbf{B})^{-1} \cdot \boldsymbol{\rho}^{\alpha} \frac{\partial}{\partial q^{\alpha}}+\mathbf{n} \frac{\partial}{\partial z}=(\mathbf{A}-z \mathbf{B})^{-1} \cdot \nabla_{S}+\mathbf{n} \frac{\partial}{\partial z}, \\
& \nabla_{S}^{ \pm}=\left(\mathbf{A} \mp \frac{h}{2} \mathbf{B}\right)^{-1} \cdot \boldsymbol{\rho}^{\alpha} \frac{\partial}{\partial q^{\alpha}}=\left(\mathbf{A} \mp \frac{h}{2} \mathbf{B}\right)^{-1} \cdot \nabla_{S} .
\end{aligned}
$$

We note that the inverse of the tensor $\mathbf{A}-z \mathbf{B}$ is understood here and henceforth as the inverse of a two-dimensional tensor, i.e.,

$$
(\mathbf{A}-z \mathbf{B})^{-1} \cdot(\mathbf{A}-z \mathbf{B})=(\mathbf{A}-z \mathbf{B}) \cdot(\mathbf{A}-z \mathbf{B})^{-1}=\mathbf{A} .
$$

We assume that the surface stresses act on the face surfaces $\Omega_{ \pm}$, i.e., $\Omega_{2}=\Omega_{+} \cup \Omega_{-}$. This leads to the following boundary conditions on $\Omega_{ \pm}$:

$$
\left.\left(\mathbf{n}_{ \pm} \cdot \boldsymbol{\sigma} \mp \nabla_{S}^{ \pm} \cdot \tau_{S}^{ \pm}\right)\right|_{\Omega_{ \pm}}=\varphi_{ \pm} .
$$

In (2.1), $\mathbf{n}_{ \pm}$are normal vectors to $\Omega_{ \pm}$(see Fig. 1), $\boldsymbol{\tau}_{S}^{ \pm}$and $\boldsymbol{\varphi}_{ \pm}$are the respective surface stresses and surface loads acting on $\Omega_{ \pm}$:

$$
\boldsymbol{\tau}_{ \pm}=\tau_{0}^{ \pm} \mathbf{A}+\lambda_{S}^{ \pm} \mathbf{A} \operatorname{tr} \boldsymbol{\epsilon}_{ \pm}+2 \mu_{S}^{ \pm} \boldsymbol{\epsilon}_{ \pm}, \quad 2 \boldsymbol{\epsilon}_{ \pm}=\left(\nabla_{S}^{ \pm} \mathbf{u}_{S}^{ \pm}\right) \cdot \mathbf{A}+\mathbf{A} \cdot\left(\nabla_{S}^{ \pm} \mathbf{u}_{S}^{ \pm}\right)^{T} .
$$

Here $\mu_{S}^{ \pm}$and $\lambda_{S}^{ \pm}$are the surface elastic moduli, and $\tau_{0}^{ \pm}$are the residual stresses on $\Omega_{ \pm}$. We note that, in general, the surface gradient operators $\nabla_{S}^{ \pm}$on $\Omega_{ \pm}$differ from each other.

To pass to two-dimensional equations of the shell theory, we integrate equilibrium equations (1.3) over the thickness with boundary conditions (2.1) taken into account. This procedure is described in detail, for example, in $[35,39,41]$. Integrating Eqs. (1.3) over $z$ with (1.2) taken into account, we obtain

$$
\begin{aligned}
& \nabla_{S} \cdot \mathbf{T}+G_{+} \nabla_{S}^{+} \cdot \boldsymbol{\tau}_{+}+G_{-} \nabla_{S}^{-} \cdot \boldsymbol{\tau}_{-}+\mathbf{q}=\mathbf{0}, \\
& \mathbf{T}=\left\langle(\mathbf{A}-z \mathbf{B})^{-1} \cdot \boldsymbol{\sigma}\right\rangle, \quad \mathbf{q}=G_{+} \boldsymbol{\varphi}_{+}-G_{-} \boldsymbol{\varphi}_{-}+\langle\mathbf{f}\rangle, \quad\langle(\ldots)\rangle=\int_{-h / 2}^{h / 2}(\ldots) G d z, \\
& G=G(z) \equiv \operatorname{det}(\mathbf{A}-z \mathbf{B}), \quad G_{ \pm}=G\left( \pm \frac{1}{2} h\right) .
\end{aligned}
$$

The quantity $\mathbf{T}$ is the stress tensor and $\mathbf{q}$ is the surface density of external forces acting on the shell.

We perform the vector multiplication of equations of equilibrium (1.3) from the left by $z \mathbf{n}$ and integrate over the thickness with the boundary conditions (2.1) taken into account to obtain the second equilibrium equation

$$
\nabla_{S} \cdot \mathbf{M}+\mathbf{T}_{\times}+\mathbf{m}+\frac{h}{2} G_{+} \mathbf{n} \times \nabla_{S}^{+} \cdot \boldsymbol{\tau}_{+}-\frac{h}{2} G_{-} \mathbf{n} \times \nabla_{S}^{-} \cdot \boldsymbol{\tau}_{-}=\mathbf{0},
$$




$$
\mathbf{M}=-\left\langle(\mathbf{A}-z \mathbf{B})^{-1} \cdot z \boldsymbol{\sigma} \times \mathbf{n}\right\rangle, \quad \mathbf{m}=\frac{h}{2} G_{+} \mathbf{n} \times \boldsymbol{\varphi}_{+}+\frac{h}{2} G_{-} \mathbf{n} \times \boldsymbol{\varphi}_{-}+\langle z \mathbf{n} \times \mathbf{f}\rangle
$$

The tensor $\mathbf{M}$ is the moment tensor and $\mathbf{m}$ is the surface moment distributed over $\omega$; the subscript $\times$ denotes a vector invariant of a second-rank tensor, which was introduced by J. W. Gibbs [42] (also see $[41,43,44])$. For the dyad formed by vectors $\mathbf{a}$ and $\mathbf{b}$, the vector invariant is calculated by the formula $(\mathbf{a} \otimes \mathbf{b})_{\times}=\mathbf{a} \times \mathbf{b}$, which extends to second-rank tensors by linearity.

The presence of terms related to $\boldsymbol{\tau}_{ \pm}$distinguishes equilibrium equations (2.2) and (2.4) from the equilibrium equations of the linear theory of shells $[23,33,35,40]$. Let us simplify them. We have the relation that follows from the Hamilton-Cayley theorem for two-dimensional tensors:

$$
G(\mathbf{A}-z \mathbf{B})^{-1}=\mathbf{A}+z(\mathbf{B}-\mathbf{A} \operatorname{tr} \mathbf{B}) .
$$

This relation implies the identity

$$
G_{ \pm} \nabla_{S}^{ \pm}=\nabla_{S} \pm \frac{h}{2}(\mathbf{B}-\mathbf{A} \operatorname{tr} \mathbf{B}) \cdot \nabla_{S}
$$

which permits writing the terms depending on $\boldsymbol{\tau}_{ \pm}$as

$$
\begin{aligned}
& G_{+} \nabla_{S}^{+} \cdot \boldsymbol{\tau}_{+}+G_{-} \nabla_{S}^{-} \cdot \boldsymbol{\tau}_{-}=\nabla_{S} \cdot\left(\boldsymbol{\tau}_{+}+\boldsymbol{\tau}_{-}\right)+O(h\|\mathbf{B}\|), \\
& \begin{array}{c}
G_{+} \mathbf{n} \times \nabla_{S}^{+} \cdot \boldsymbol{\tau}_{+}-G_{-} \mathbf{n} \times \nabla_{S}^{-} \cdot \boldsymbol{\tau}_{-}=\mathbf{n} \times \nabla_{S} \cdot\left(\boldsymbol{\tau}_{+}-\boldsymbol{\tau}_{-}\right)+O(h\|\mathbf{B}\|) \\
\quad=-\nabla_{S} \cdot\left[\left(\boldsymbol{\tau}_{+}-\boldsymbol{\tau}_{-}\right) \times \mathbf{n}\right]+\left[\left(\boldsymbol{\tau}_{+}-\boldsymbol{\tau}_{-}\right) \cdot \mathbf{B}\right]_{\times}+O(h\|\mathbf{B}\|) .
\end{array}
\end{aligned}
$$

Here the terms of the order of $O(h\|\mathbf{B}\|)$ compared to the other terms are usually neglected in the linear theory of shells. Further, we assume that $h\|\mathbf{B}\| \ll 1$. From the physical viewpoint, this means that the shell is thin and its surface $\omega$ is described by sufficiently smooth functions. Thus, in Eqs. (2.2) and (2.4) and further, we use the relations $G_{ \pm}=1$ and $\nabla_{S}^{ \pm}=\nabla_{S}$.

This assumption permits, instead of (2.3) and (2.5), using simpler formulas for stresses and moments, which were also used in [38-40]: $\mathbf{T}=\langle\mathbf{A} \cdot \boldsymbol{\sigma}\rangle$ and $\mathbf{M}=-\langle\mathbf{A} \cdot z \boldsymbol{\sigma} \times \mathbf{n}\rangle$. Thus, Eqs. (2.2) and (2.4) become

$$
\begin{aligned}
& \nabla_{S} \cdot \mathbf{T}+\nabla_{S} \cdot\left(\boldsymbol{\tau}_{+}+\boldsymbol{\tau}_{-}\right)+\mathbf{q}=\mathbf{0}, \\
& \nabla_{S} \cdot \mathbf{M}-\frac{h}{2} \nabla_{S} \cdot\left[\left(\boldsymbol{\tau}_{+}-\boldsymbol{\tau}_{-}\right) \times \mathbf{n}\right]+\mathbf{T}_{\times}+\left[\left(\boldsymbol{\tau}_{+}-\boldsymbol{\tau}_{-}\right) \cdot \mathbf{B}\right]_{\times}+\mathbf{m}=\mathbf{0} .
\end{aligned}
$$

Since $\mathbf{m} \cdot \mathbf{n}=0$, Eq. (2.7) implies the "sixth equation of equilibrium" in algebraic form:

$$
\mathbf{M} \cdot \mathbf{B}+\mathbf{T}_{\times} \cdot \mathbf{n}=0 .
$$

Equations (2.6) and (2.7) permit introducing effective stress and moment tensors $\mathbf{T}^{*}$ and $\mathbf{M}^{*}$ :

$$
\begin{aligned}
& \mathbf{T}^{*}=\mathbf{T}+\mathbf{T}_{S}, \quad \mathbf{M}^{*}=\mathbf{M}+\mathbf{M}_{S}, \\
& \mathbf{T}_{S}=\boldsymbol{\tau}_{+}+\boldsymbol{\tau}_{-}, \quad \mathbf{M}_{S}=-\frac{h}{2}\left(\boldsymbol{\tau}_{+}-\boldsymbol{\tau}_{-}\right) \times \mathbf{n} .
\end{aligned}
$$

To describe the shell strains, we assume the displacement field to be linear in thickness; this approximation is used in the theory of plates and shells with the transverse shear taken into account [35, 45, 49]:

$$
\mathbf{u}\left(q^{1}, q^{2}, z\right)=\mathbf{w}\left(q^{1}, q^{2}\right)-z \boldsymbol{\vartheta}\left(q^{1}, q^{2}\right), \quad \mathbf{n} \cdot \boldsymbol{\vartheta}=0 .
$$

Here we assume that the rotation vector $\boldsymbol{\vartheta}$ is kinematically independent of the vector of the shell median surface displacements w. Formula (2.9) implies

$$
\begin{aligned}
& \mathbf{u}_{S}^{ \pm}=\mathbf{w} \mp \frac{h}{2} \boldsymbol{\vartheta}, \quad \boldsymbol{\epsilon}_{ \pm}=\boldsymbol{\epsilon} \mp \frac{h}{2} \boldsymbol{\kappa}, \\
& \boldsymbol{\epsilon}=\frac{1}{2}\left[\nabla_{S} \mathbf{w} \cdot \mathbf{A}+\mathbf{A} \cdot\left(\nabla_{S} \mathbf{w}\right)^{T}\right], \quad \boldsymbol{\kappa}=\frac{1}{2}\left[\nabla_{S} \boldsymbol{\vartheta} \cdot \mathbf{A}+\mathbf{A} \cdot\left(\nabla_{S} \boldsymbol{\vartheta}\right)^{T}\right],
\end{aligned}
$$

where $\boldsymbol{\epsilon}$ and $\boldsymbol{\kappa}$ are two-dimensional tensors of tensile-shear and bending-torsion strains. We use (2.10) to obtain the expressions for the surface stresses $\boldsymbol{\tau}_{ \pm}$:

$$
\boldsymbol{\tau}_{ \pm}=\tau_{0}^{ \pm} \mathbf{A}+\lambda_{ \pm}^{S} \mathbf{A} \operatorname{tr} \boldsymbol{\epsilon}+2 \mu_{ \pm}^{S} \boldsymbol{\epsilon} \mp \frac{h}{2}\left(\lambda_{ \pm}^{S} \mathbf{A} \operatorname{tr} \boldsymbol{\kappa}+2 \mu_{ \pm}^{S} \boldsymbol{\kappa}\right) .
$$


Thus, we have the formulas

$$
\begin{aligned}
& \boldsymbol{\tau}_{+}+\boldsymbol{\tau}_{-}=\left(\tau_{0}^{+}+\tau_{0}^{-}\right) \mathbf{A}+\left(\lambda_{+}^{S}+\lambda_{-}^{S}\right) \mathbf{A} \operatorname{tr} \boldsymbol{\epsilon}+2\left(\mu_{+}^{S}+\mu_{-}^{S}\right) \boldsymbol{\epsilon}-\frac{h}{2}\left[\left(\lambda_{+}^{S}-\lambda_{-}^{S}\right) \mathbf{A} \operatorname{tr} \boldsymbol{\kappa}+2\left(\mu_{+}^{S}-\mu_{-}^{S}\right) \boldsymbol{\kappa}\right], \\
& \boldsymbol{\tau}_{+}-\boldsymbol{\tau}_{-}=\left(\tau_{0}^{+}+\tau_{0}^{-}\right) \mathbf{A}+\left(\lambda_{+}^{S}+\lambda_{-}^{S}\right) \mathbf{A} \operatorname{tr} \boldsymbol{\epsilon}+2\left(\mu_{+}^{S}-\mu_{-}^{S}\right) \boldsymbol{\epsilon}-\frac{h}{2}\left[\left(\lambda_{+}^{S}+\lambda_{-}^{S}\right) \mathbf{A} \operatorname{tr} \boldsymbol{\kappa}+2\left(\mu_{+}^{S}-\mu_{-}^{S}\right) \boldsymbol{\kappa}\right] .
\end{aligned}
$$

In the case of shells with equal surface properties, i.e., in the case where $\tau_{0}^{+}=\tau_{0}^{-}=\tau_{0}, \mu_{+}^{S}=\mu_{-}^{S}=\mu_{S}$, and $\lambda_{+}^{S}=\lambda_{-}^{S}=\lambda_{S}$, these formulas simplify:

$$
\begin{aligned}
& \boldsymbol{\tau}_{+}+\boldsymbol{\tau}_{-}=2 \tau_{0} \mathbf{A}+2 \lambda_{S} \mathbf{A} \operatorname{tr} \boldsymbol{\epsilon}+4 \mu_{S} \boldsymbol{\epsilon}, \\
& \boldsymbol{\tau}_{+}-\boldsymbol{\tau}_{-}=-h\left(\lambda_{S} \mathbf{A} \operatorname{tr} \boldsymbol{\kappa}+2 \mu_{S} \boldsymbol{\kappa}\right) .
\end{aligned}
$$

This implies that the stress and moment tensors generated by the action of surface stresses are equal to

$$
\begin{aligned}
& \mathbf{T}_{S}=2 \tau_{0} \mathbf{A}+C_{1}^{S} \boldsymbol{\epsilon}+C_{2}^{S} \mathbf{A} \operatorname{tr} \boldsymbol{\epsilon}, \quad \mathbf{M}_{S}=-\left(D_{1}^{S} \boldsymbol{\kappa}+D_{2}^{S} \mathbf{A} \operatorname{tr} \boldsymbol{\kappa}\right) \times \mathbf{n}, \\
& C_{1}^{S}=4 \mu^{S}, \quad C_{2}^{S}=2 \lambda^{S}, \quad D_{1}^{S}=h^{2} \mu^{S}, \quad D_{2}^{S}=D \nu+\frac{h^{2} \lambda^{S}}{2}
\end{aligned}
$$

With (2.8) taken into account, it follows from (2.11) that the surface stresses do not affect the cutting forces, because $\mathbf{T}_{S} \cdot \mathbf{n}=\mathbf{0}$, and do not affect the transverse shear stiffness of the shell. It also follows from (2.11) that the residual surface stresses $\tau_{0}$ do not affect the shell stiffness, although they naturally affect its stressed state.

For $\mathbf{T}$ and $\mathbf{M}$ we take the equations of state in the simplest form, which are presented, for example, in [35]:

$$
\begin{aligned}
& \mathbf{T} \cdot \mathbf{A}-\frac{1}{2}(\mathbf{M} \cdot \mathbf{B}) \mathbf{A} \times \mathbf{n}=\frac{\partial W_{S}}{\partial \boldsymbol{\epsilon}}, \quad \mathbf{T} \cdot \mathbf{n}=\frac{\partial W_{S}}{\partial \gamma}, \quad \mathbf{M}=\frac{\partial W_{S}}{\partial \boldsymbol{\kappa}}, \\
& 2 W_{S}=\boldsymbol{\epsilon} \cdot \mathbf{C} \cdot \boldsymbol{\epsilon}+\boldsymbol{\kappa} \cdot \mathbf{D} \cdot \boldsymbol{\kappa}+\Gamma \boldsymbol{\gamma} \cdot \boldsymbol{\gamma} .
\end{aligned}
$$

Here $W_{S}$ is the potential strain energy of the shell, $\mathbf{C}$ and $\mathbf{D}$ are fourth-rank tensors determining the shell tangential and flexural stiffnesses, $\boldsymbol{\gamma}$ is the transverse shear vector: $\boldsymbol{\gamma}=\nabla_{S}(\mathbf{w} \cdot \mathbf{n})-\boldsymbol{\vartheta}$, and $\Gamma$ is the transverse shear stiffness. For isotropic shells, $\mathbf{C}$ and $\mathbf{D}$ are expressed as [35]:

$$
\begin{aligned}
& \mathbf{C}=C_{11} \mathbf{a}_{1} \mathbf{a}_{1}+C_{22}\left(\mathbf{a}_{2} \mathbf{a}_{2}+\mathbf{a}_{2} \mathbf{a}_{4}\right), \quad \mathbf{D}=D_{22}\left(\mathbf{a}_{2} \mathbf{a}_{2}+\mathbf{a}_{4} \mathbf{a}_{4}\right)+D_{33} \mathbf{a}_{3} \mathbf{a}_{3}, \\
& \mathbf{a}_{1}=\mathbf{A} \equiv \mathbf{e}_{1} \otimes \mathbf{e}_{1}+\mathbf{e}_{2} \otimes \mathbf{e}_{2}, \quad \mathbf{a}_{2}=\mathbf{e}_{1} \otimes \mathbf{e}_{1}-\mathbf{e}_{2} \otimes \mathbf{e}_{2}, \\
& \mathbf{a}_{3}=-\mathbf{A} \times \mathbf{n}=\mathbf{e}_{1} \otimes \mathbf{e}_{2}-\mathbf{e}_{2} \otimes \mathbf{e}_{1}, \quad \mathbf{a}_{4}=\mathbf{e}_{1} \otimes \mathbf{e}_{2}+\mathbf{e}_{2} \otimes \mathbf{e}_{1},
\end{aligned}
$$

where $\mathbf{e}_{1}, \mathbf{e}_{2}$ are unit vectors lying in the tangent plane to $\omega\left(\mathbf{e}_{1} \cdot \mathbf{e}_{2}=\mathbf{e}_{1} \cdot \mathbf{n}=\mathbf{e}_{2} \cdot \mathbf{n}=0\right)$. The components $C_{11}, C_{22}, D_{22}, D_{33}$, and $\Gamma$ are given by the formulas

$$
\begin{aligned}
& C_{11}=\frac{E h}{2(1-\nu)}, \quad C_{22}=\frac{E h}{2(1+\nu)}, \quad D_{22}=\frac{E h^{3}}{24(1+\nu)}, \quad D_{33}=\frac{E h^{3}}{24(1-\nu)}, \quad \Gamma=k \mu h, \\
& E=2 \mu(1+\nu), \quad \nu=\frac{\lambda}{2(\lambda+\mu)}, \quad C \equiv C_{11}+C_{22}=\frac{E h}{1-\nu^{2}}, \quad D \equiv D_{11}+D_{22}=\frac{E h^{3}}{12\left(1-\nu^{2}\right)},
\end{aligned}
$$

where $C$ and $D$ are the shell tangential and flexural stiffnesses, $E$ and $\nu$ are Young's modulus and Poisson's ratio of the shell material, and $k$ is the transverse shear coefficient [35, 45].

\section{THEORY OF PLATES}

The equations of state have the simplest form in the case of plate. Here $\mathbf{B}=\mathbf{0}$ and $\mathbf{n}=\mathbf{i}_{3}$. The effective stresses and moments are expressed as [32]:

$$
\begin{aligned}
& \mathbf{T}^{*}=C_{1} \boldsymbol{\epsilon}+C_{2} \mathbf{A} \operatorname{tr} \boldsymbol{\epsilon}+\Gamma \boldsymbol{\gamma} \otimes \mathbf{i}_{3}, \quad \mathbf{M}^{*}=-\left(D_{1} \boldsymbol{\kappa}+D_{2} \mathbf{A} \operatorname{tr} \boldsymbol{\kappa}\right) \times \mathbf{i}_{3}, \\
& C_{1}=C(1-\nu)+4 \mu^{S}, \quad C_{2}=C \nu+2 \lambda^{S}, \quad D_{1}=D(1-\nu)+h^{2} \mu^{S}, \quad D_{2}=D \nu+\frac{h^{2} \lambda^{S}}{2} .
\end{aligned}
$$

The plate effective tangential and flexural stiffnesses are equal to

$$
C_{\text {eff }} \equiv C_{1}+C_{2}=C+4 \mu_{S}+2 \lambda_{S}, \quad D_{\text {eff }} \equiv D_{1}+D_{2}=D+h^{2} \mu_{S}+\frac{h^{2} \lambda^{S}}{2} .
$$




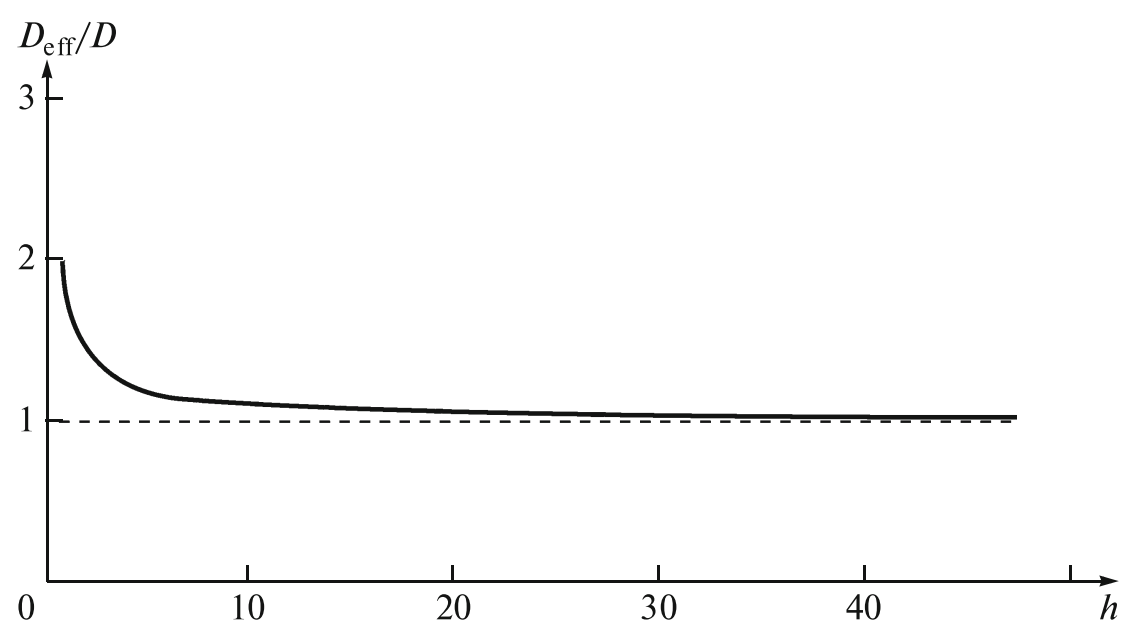

Fig. 2.

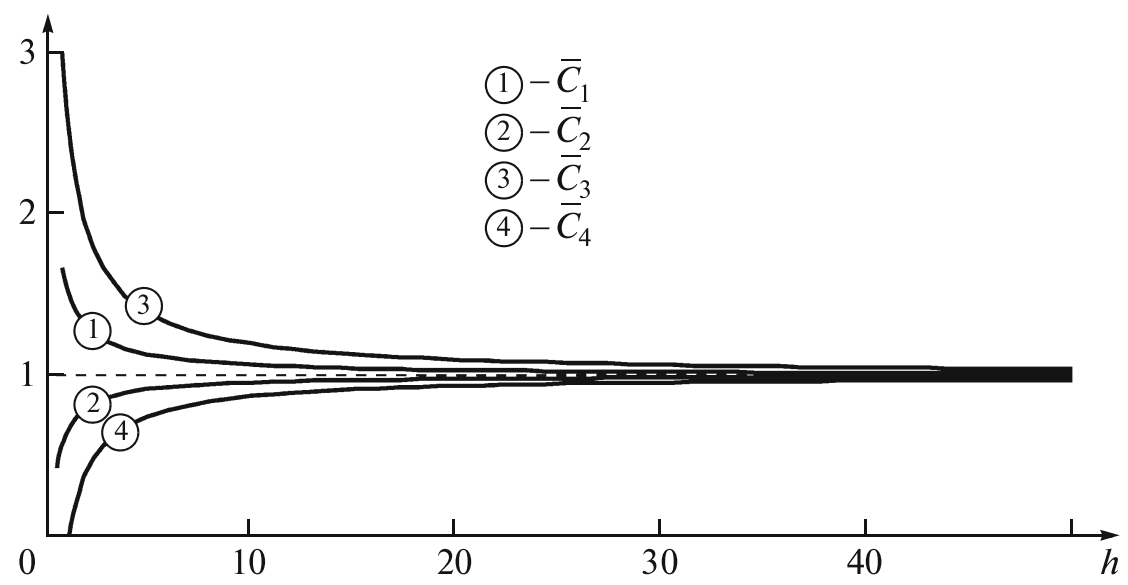

Fig. 3 .

The equations of state (3.1) permit writing the plate equilibrium equations with the surface stresses (2.6) and (2.7) taken into account in terms of displacements $\mathbf{w}$ and rotations $\boldsymbol{\vartheta}$. In particular, the equation for the deflection $w=\mathbf{w} \cdot \mathbf{i}_{3}$ can be reduced to the form [36]:

$$
D_{\text {eff }} \Delta \Delta w=\nabla_{S} \cdot \mathbf{m}-\frac{D_{\text {eff }}}{\Gamma} \Delta q_{n}+q_{n}, \quad q_{n}=\mathbf{q} \cdot \mathbf{i}_{3}, \quad \Delta=\nabla_{S} \cdot \nabla_{S} .
$$

We note that the equations of state for effective stresses and moments (3.1) qualitatively differ from the equations of state for isotropic homogeneous plates. The dependence of $\mathbf{T}$ and $\mathbf{M}$ on strains is determined by the four elastic constants: $C, D, \nu, \Gamma$. The tensors $\mathbf{T}^{*}$ and $\mathbf{M}^{*}$ are determined by five independent elastic constants: $C_{1}, C_{2}, D_{1}, D_{2}, \Gamma$.

To obtain quantitative estimates of the results of the surface stresses influence, we use the data for aluminum obtained by simulation methods in [29] and also used in [1, 3, 4]. For the bulk phase, we set $\mu=34.7 \mathrm{GPa}$ and $\nu=0.3$. For $\lambda^{S}$ and $\mu^{S}$ we take the following values: $\lambda^{S}=-3.48912 \mathrm{~N} / \mathrm{m}$ and $\mu^{S}=6.2178 \mathrm{~N} / \mathrm{m}$.

Let us have a look at how $D_{\text {eff }}, C_{1}, C_{2}, D_{1}$, and $D_{2}$ depend on the thickness $h$. The dependence of the flexural stiffness $D_{\text {eff }}$ on the thickness is displayed in Fig. 2. The graphs of dimensionless quantities $\bar{C}_{1}=C_{1} /[C(1-\nu)], \bar{C}_{2}=C_{2} /(C \nu), \bar{D}_{1}=D_{1} /[D(1-\nu)], \bar{D}_{2}=D_{2} /(D \nu)$ are shown in Fig. 3, curves 1 to 4 .

One can see from Figs. 2 and 3 that for $h>50 \mathrm{~nm}$, the influence of surface stresses is practically unnoticeable. Their influence is most significant for $h<20 \mathrm{~nm}$. In addition, one can see that the surface stresses affect the stiffness differently - some of them increase $\left(C_{1}\right.$ and $\left.D_{1}\right)$, while others $\left(C_{2}\right.$ 


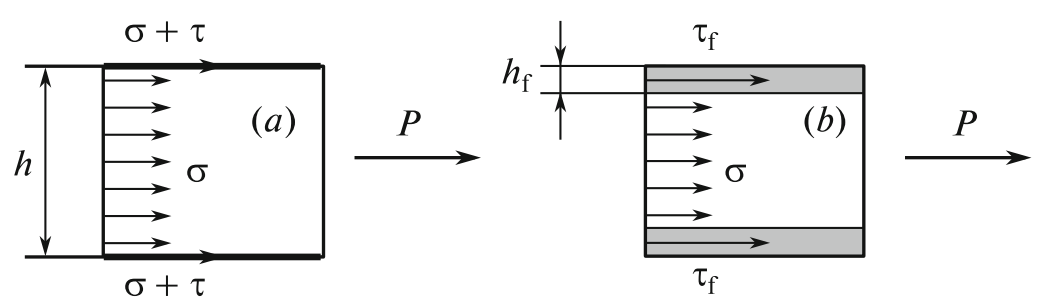

Fig. 4.

and $D_{2}$ ) decrease, and even take zero values for some values of thickness. Nevertheless, we note that

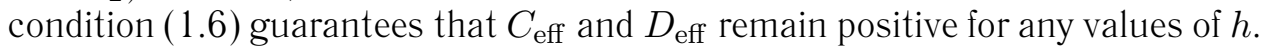

\section{COMPARISON WITH THE THEORY OF THREE-LAYER PLATES}

The above concept of surface stresses is based on the assumption that the surface layer properties differ from the properties of the material in the bulk. In other words, the equations of state for the near-surface stresses must differ from the equations of state in the bulk. Methods for studying inhomogeneities with respect to thickness, for example, in the case of three-layer plates and shells, were developed in the theory of plates and shells (e.g., see [35, 46-49]). It is easy to see that there is a certain similarity between the theory with surface stresses and the theory of three-layer plates and shells. First, we consider the simplest problem on tension by a force $P$ of a strip with surface tensions (Fig. $4 a$ ) and a three-layer strip of symmetric structure (Fig. $4 b$ ). If we denote the stress in the bulk by $\sigma$, the surface stress by $\tau$, and the stress in the surface layer by $\tau_{\mathrm{f}}$, then we obtain the relations $P=\sigma h+2 \tau$ and $P=\sigma\left(h-2 h_{\mathrm{f}}\right)+2 \tau_{\mathrm{f}} h_{\mathrm{f}}$, where $h$ and $h_{\mathrm{f}}$ are the total thickness and the surface layer thickness, respectively. It follows that

$$
\tau=\left(\tau_{\mathrm{f}}-\sigma\right) h_{\mathrm{f}} .
$$

The relation permits interpreting the surface stress $\tau$ as an excessive (compared to that in the bulk) force acting in a surface layer of thickness $f_{\mathrm{f}}$. The case with surface stresses is obtained as the limit case as $h_{\mathrm{f}} \rightarrow 0$ :

$$
\tau=\lim _{h_{\mathrm{f}} \rightarrow 0} \tau_{\mathrm{f}} h_{\mathrm{f}}
$$

In addition, this simple example permits estimating the advantages of the surface stress concept compared with the three-layer model, which originate from experimental studies of nanodimensional plates. To determine $\tau$, it suffices to perform fewer experiments. The point is that the surface layer thickness $h_{\mathrm{f}}$ is generally unknown, and the stress distribution in the layer is hardly homogeneous. Actually, the concept of surface stresses implies the use of the direct approach to the surface stress modeling, i.e., the construction of two-dimensional equations of state for the force tensor $\boldsymbol{\tau}$ instead of three-dimensional equations for the surface layer with their subsequent averaging over the thickness.

To analyze the similarity and distinctions between the plate model with surface stresses and the three-layer plate in more detail, we use the method for determining effective stiffnesses of three-layer plates [33-36]. We consider an isotropic plate with a symmetric cross-section with respect to the thickness (Fig. $4 b$ ). Let $h_{\mathrm{c}}$ be the thickness of the internal layer (kernel), and let $h_{\mathrm{f}}$ be the thickness of the surface layers, and $h_{\mathrm{c}} \gg h_{\mathrm{f}}$. The total thickness is equal to $h=h_{\mathrm{c}}+2 h_{\mathrm{f}}$. The kernel and surface layer material properties are given by Young's moduli $E_{\mathrm{c}}$ and $E_{\mathrm{f}}$ and Poisson's ratios $\nu_{\mathrm{c}}$ and $\nu_{\mathrm{f}}$ (or the shear moduli $\mu_{\mathrm{c}}$ and $\mu_{\mathrm{f}}$ ).

The equations of state for the three-layer plate have the form [34-36]:

$$
\mathbf{T}=C_{1} \boldsymbol{\epsilon}+C_{2} \mathbf{A} \operatorname{tr} \boldsymbol{\epsilon}+\Gamma \boldsymbol{\gamma} \otimes \mathbf{i}_{3}, \quad \mathbf{M}=-\left(D_{1} \boldsymbol{\kappa}+D_{2} \mathbf{A} \operatorname{tr} \boldsymbol{\kappa}\right) \times \mathbf{i}_{3},
$$

where the effective stiffnesses are equal to

$$
\begin{array}{ll}
C_{1}=2 C_{22}, \quad C_{2}=C_{11}-C_{22}, & D_{1}=2 D_{22}, \quad D_{2}=D_{33}-D_{22}, \quad \Gamma=\ell^{2} D_{22}, \\
C_{11}=\frac{1}{2}\left(\frac{2 E_{\mathrm{f}} h_{\mathrm{f}}}{1-\nu_{\mathrm{f}}}+\frac{E_{\mathrm{c}} h_{\mathrm{c}}}{1-\nu_{\mathrm{c}}}\right), & C_{22}=\frac{1}{2}\left(\frac{2 E_{\mathrm{f}} h_{\mathrm{f}}}{1-\nu_{\mathrm{f}}}+\frac{E_{\mathrm{c}} h_{\mathrm{c}}}{1+\nu_{\mathrm{c}}}\right),
\end{array}
$$




$$
D_{22}=\frac{1}{24}\left[\frac{E_{\mathrm{f}}\left(h^{3}-h_{\mathrm{c}}^{3}\right)}{1+\nu_{\mathrm{f}}}+\frac{E_{\mathrm{c}} h_{\mathrm{c}}^{3}}{1+\nu_{\mathrm{c}}}\right], \quad D_{33}=\frac{1}{24}\left[\frac{E_{\mathrm{f}}\left(h^{3}-h_{\mathrm{c}}^{3}\right)}{1-\nu_{\mathrm{f}}}+\frac{E_{\mathrm{c}} h_{\mathrm{c}}^{3}}{1-\nu_{\mathrm{c}}}\right]
$$

and $\ell$ is the least positive root of the equation

$$
\mu_{0} \cos \ell \frac{h_{\mathrm{f}}}{2} \cos \ell \frac{h_{\mathrm{c}}}{2}-\sin \ell \frac{h_{\mathrm{f}}}{2} \sin \ell \frac{h_{\mathrm{c}}}{2}=0, \quad \mu_{0}=\frac{\mu_{\mathrm{c}}}{\mu_{\mathrm{f}}} .
$$

The flexural stiffness of the three-layer plate is equal to

$$
D_{\text {eff }}=D_{33}+D_{44}=\frac{1}{12}\left[\frac{E_{\mathrm{f}}\left(h^{3}-h_{\mathrm{c}}^{3}\right)}{1-\nu_{\mathrm{f}}^{2}}+\frac{E_{\mathrm{c}} h_{\mathrm{c}}^{3}}{1-\nu_{\mathrm{c}}^{2}}\right] .
$$

Comparing the equations of state with the surface stresses taken into account (3.1) and relations (4.1), we can interpret the surface moduli $\lambda_{S}$ and $\mu_{S}$ in terms of the elastic characteristics of the surface layer $E_{\mathrm{f}}$ and $\nu_{\mathrm{f}}$ and its thickness $h_{\mathrm{f}}$. Assuming that $E_{\mathrm{c}}=E$ and $\nu_{\mathrm{c}}=\nu$ and comparing the tangential stiffnesses as $h_{\mathrm{f}} \rightarrow 0$, we obtain up to $O\left(h_{\mathrm{f}}^{2}\right)$ :

$$
\mu_{S} \approx \frac{E_{\mathrm{f}} h_{\mathrm{f}}}{2\left(1+\nu_{\mathrm{f}}\right)} \equiv \mu_{\mathrm{f}} h_{\mathrm{f}}, \quad \lambda_{S} \approx \frac{\nu_{\mathrm{f}} E_{\mathrm{f}} h_{\mathrm{f}}}{1-\nu_{\mathrm{f}}^{2}} \equiv \lambda_{\mathrm{f}} h_{\mathrm{f}} \frac{1-2 \nu_{\mathrm{f}}}{1-\nu_{\mathrm{f}}},
$$

where $\lambda_{\mathrm{f}}$ is the elastic Lamé constant of the surface layer. The same formulas are obtained by comparing the flexural stiffnesses as $h_{\mathrm{f}} \rightarrow 0$. Thus, we obtain the limit relations

$$
\mu_{S}=\lim _{h_{\mathrm{f}} \rightarrow 0} \mu_{\mathrm{f}} h_{\mathrm{f}}, \quad \lambda_{S}=\lim _{h_{\mathrm{f}} \rightarrow 0} \lambda_{\mathrm{f}} \frac{1-2 \nu_{\mathrm{f}}}{1-\nu_{\mathrm{f}}} h_{\mathrm{f}} .
$$

These relations permit interpreting the surface elastic moduli $\mu_{S}$ and $\lambda_{S}$ in terms of the Lamé constants of the surface layer and its thickness. At the same time, we note that the formulas for the stiffness of three-layer plates (4.1) were obtained under the assumption that the layer material properties are homogeneous. In the case of nano shells and plates, this condition is generally difficult to verify, and it is hardly satisfied. It is also possible to perform the comparison with the theory of functionally gradient plates and shells, which is presented, for example, in [36, 49, 50]. Prescribing a certain law of the material properties distribution in the surface layer, we can obtain formulas relating the surface moduli to the surface layer properties.

Formulas (4.2) are asymptotically exact as $h_{\mathrm{f}} \rightarrow 0$. As was already noted, for finite values of $h_{\mathrm{f}}$, they hold up to terms of the order of $O\left(h_{\mathrm{f}}^{2}\right)$. If we take these terms and the terms of greater order of magnitude into account, then it is easy to verify that the comparison of tangential and flexural stiffnesses given by formulas (3.1) and (4.1) leads to different expressions for $\mu_{S}$ and $\lambda_{S}$ depending on whether the tangential or flexural stiffness are compared. Naturally, this difference is quantitatively small, but theoretically, it permits concluding that the theory of plates with surface stresses taken into account is equivalent to the theory of three-layer plates only asymptotically and cannot be reduced to it.

\section{CONCLUSION}

We obtained two-dimensional equations of equilibrium for plates and shells with the transverse shear and surface stresses taken into account. We presented relations for stress and moment tensors and found expressions for the shell effective stiffnesses. In particular, it was shown that, with the surface stresses taken into account, the plate stiffnesses vary significantly, which agrees with the results of theoretical analysis and with experimental data given in the literature (e.g., see review [1]). In particular, it was shown that the shell flexural stiffness significantly increases for thicknesses in the nanorange. The above comparison with the case of three-layer plates showed that taking the surface stresses into account is equivalent to the existence of surface layers of a certain thickness, and in this case, the surface elastic moduli are expressed in terms of the Lamé constants of the surface layer material multiplied by its thickness. At the same time, this comparison shows the distinctions from the case of three-layer shells. These two theories coincide up to $O\left(h_{\mathrm{f}}^{2}\right)$. Moreover, the surface stresses do not affect the transverse shear stiffness.

The introduction of surface stresses corresponds to the direct approach, i.e., to the formulation of two-dimensional equations of state for the body surface. In the case of mechanics of nanodimensional 
plates and shells with the surface stresses, it seems logical to use the direct approach for constructing the theory of plates and shells on the whole, i.e., to introduce the constitutive relations for the shell as for a two-dimensional continuum without separating the terms corresponding to bulk and surface properties. Moreover, in the direct approach, there is no need for approximations of the type (2.9), integration of the equations of equilibrium or motion of a three-dimensions shell body over the thickness, and asymptotic methods and other procedures used in the theory of plates and shells (e.g., see $[23,35,39,40,47,49,51-55])$. A certain drawback of the direct approach is that it is necessary to determine the material constants contained in the equations of state theoretically and/or experimentally, which can be a complicated problem. Here this drawback is removed to a certain extent by the fact that it is necessary to find the surface constants $\lambda_{S}$ and $\mu_{S}$ along with $\lambda$ and $\mu$ independently.

\section{ACKNOWLEDGMENTS}

The authors thank E. A. Ivanova for discussions of the results of this paper.

The research was financially supported by the Russian Foundation for Basic Research (project No. 09-01-00459) and by DFG (Grant No. AL 341/31-1).

\section{REFERENCES}

1. H. L. Duan, J. Wang, and B. L. Karihaloo, "Theory of Elasticity at the Nanoscale," Adv. Appl. Mech. 42, $1-63(2008)$.

2. L. D. Landau and E. M. Lifshitz, Course of Theoretical Physics, Vol. 6: Hydrodynamics (Nauka, Moscow, 1986) [in Russian].

3. J. Wang, H. L. Duan, Z. P. Huang, and B. L. Karihaloo, "A Scaling Law for Properties of Nano-Structured Materials," Proc. Roy. Soc. London. Ser. A 462 (2069), 1355-1363 (2006).

4. H. L. Duan, J. Wang, Z. P. Huang, and B. L. Karihaloo, "Size-Dependent Effective Elastic Constants of Solids Containing Nano-Inhomogeneities with Interface Stress,” J. Mech. Phys. Solids 53 (7), 1574-1596 (2005).

5. S. Cuenot, C. Frétigny, S. Demoustier-Champagne, and B. Nysten, "Surface Tension Effect on the Mechanical Properties of Nanomaterials Measured by Atomic Force Microscopy,” Phys. Rev. B 69 (16), 165410-5 (2004).

6. G. Y. Jing, H. L. Duan, X. M. Sun, et al., "Surface Effects on Elastic Properties of Silver Nanowires: Contact Atomic-Force Microscopy,” Phys. Rev. B 73 (23), 235409-6 (2006).

7. C. Q. Chen, Y. Shi, Y. S. Zhang, et al., "Size Dependence of Young's Modulus in ZnO Nanowires," Phys. Rev. Lett. 96 (7), 075505-4 (2006).

8. P. S. Laplace, Sur L'action Capillaire in Supplément au Traité de Mécanique Céleste, T. 4, Livre X (Gauthier-Villars, Paris, 1805).

9. P. S. Laplace, À la Théorie de L'action Capillaire in Supplément au Traité de Mécanique Céleste, T. 4, Livre X (Gauthier-Villars, Paris, 1805).

10. T. Young, "An Essay on the Cohesion of Fluids," Phil. Trans. Roy. Soc. London 95, 65-87 (1805).

11. J. W. Gibbs, "On the Equilibrium of Heterogeneous Substances," in The Collected Works of J. Willard Gibbs (Longmans, Green, New York, 1928), pp. 55-353.

12. E. Orowan, "Surface Energy and Surface Tension in Solids and Fluids," Proc. Roy. Soc. London. Ser. A 316 (1527), 473-491 (1970).

13. Ya. S. Podstrigach and Yu. Z. Povstenko, Introduction to Mechanics of Surface Phenomena in Strained Rigid Bodies (Naukova Dumka, Kiev, 1985) [in Russian].

14. R. Finn, Equilibrium Capillary Surfaces (Springer, New York, 1986; Mir, Moscow, 1989).

15. A. I. Rusanov, "Thermodynamics of Solid Surfaces," Surf. Sci. Rep. 23 (6-8), 173-247 (1996).

16. A. I. Rusanov, "Surface Thermodynamics Revisited," Surf. Sci. Rep. 58 (5-8), 111-239 (2005).

17. M. E. Gurtin and A. I. Murdoch, "A Continuum Theory of Elastic Material Surfaces," Arch. Rational Mech. Anal. 57 (4), 291-323 (1975).

18. M. E. Gurtin and A. I. Murdoch, "Addenda to Our Paper: A Continuum Theory of Elastic Material Surfaces," Arch. Rational Mech. Anal. 59 (4), 389-390 (1975).

19. A. I. Murdoch, "A Thermodynamical Theory of Elastic Material Interfaces," Quart. J. Mech. Appl. Math. 29 (3), 245-274 (1976).

20. P. Podio-Guidugli and G. V. Caffarelli, "Surface Interaction Potentials in Elasticity," Arch. Rational Mech. Anal. 109 (4), 343-383 (1990).

21. Yu. Z. Povstenko, "Theoretical Investigation of Phenomena Caused by Heterogeneous Surface Tension in Solids," J. Mech. Phys. Solids 41 (9), 1499-1514 (1993). 
22. D. J. Steigmann and R. W. Ogden, "Elastic Surface-Substrate Interactions," Proc. Roy. Soc. London. Ser. A 455 (1982), 437-474 (1999).

23. V. A. Eremeev and L. M. Zubov, Mechanics of Elastic Shells (Nauka, Moscow, 2008) [in Russian].

24. P. Lu, L. H. He, H. P. Lee, and C. Lu, “Thin Plate Theory Including Surface Effects," Int. J. Solids Struct. 43 (16), 4631-4647 (2006).

25. Y. J. Shi, W. L. Guo, and C. Q. Ru, "Relevance of Timoshenko-Beam Model to Microtubules of Low Shear Modulus,” Phys. E: Low-Dimens. Syst. Nanostruct. 41 (2), 213-219 (2008).

26. D. W. Huang, "Size-Dependent Response of Ultra-Thin Films with Surface Effects," Int. J. Solids Struct. $45(2), 568-579(2008)$.

27. J. Peng, J. Wu, K. C. Hwang, et al., "Can a Single-Wall Carbon Nanotube be Modeled as a Thin Shell?” J. Mech. Phys. Solids 56 (6), 2213-2224 (2008).

28. K. Dahmen, S. Lehwald, and H. Ibach, "Bending of Crystalline Plates under the Influence of Surface Stress - a Finite Element Analysis," Surf. Sci. 446 (1-2), 161-173 (2000).

29. R. E. Miller and V. B. Shenoy, "Size-Dependent Elastic Properties of Nanosized Structural Elements," Nanotech. 11 (3), 139-147 (2000).

30. J. G. Guo and Y. P. Zhao, “The Size-Dependent Elastic Properties of Nanofilms with Surface Effects,” J. Appl. Phys. 98 (7), 074306-11 (2005).

31. C. F. Lu, C. W. Lim, and W. Q. Chen, "Size-Dependent Elastic Behavior of FGM Ultra-Thin Films Based on Generalized Refined Theory,” Int. J. Solids Struct. 46 (5), 1176-1185 (2009).

32. V. A. Eremeyev, H. Altenbach, and N. F. Morozov, "The Influence of Surface Tension on the Effective Stiffness of Nanosize Plates,” Dokl. Ross. Akad. Nauk 424 (5), 618-621 (2009) [Dokl. Phys. (Engl. Transl.) 54 (2), 98-100(2009)].

33. H. Altenbach and P. A. Zhilin, "General Theory of Elastic Simple Shells," Uspekhi Mekh. 11 (4), $107-148$ (1988).

34. H. Altenbach, "An Alternative Determination of Transverse Shear Stiffness for Sandwich and Laminated Plates," Int. J. Solids Struct. 37 (25), 3503-3520 (2000).

35. P. A. Zhilin, Applied Mechanics. Foundations of Shell Theory (Izd-vo Polytechn. Univ., St. Petersburg, 2006) [in Russian].

36. H. Altenbach and V. A. Eremeyev, "Direct Approach Based Analysis of Plates Composed of Functionally Graded Materials,” Arch. Appl. Mech. 78 (10), 775-794 (2008).

37. L. M. Zubov, Methods of Nonlinear Elasticity in Shell Theory (Izd-vo RGU, Rostov-on-Don, 1982) [in Russian].

38. S. P. Timoshenko and S. Woinowsky-Krieger, Theory of Plates and Shells (McGraw Hill, New York, 1959; Fizmatgiz, Moscow, 1963).

39. A. L. Goldenveizer, Theory of Thin Elastic Shells (Nauka, Moscow, 1976) [in Russian].

40. V. V. Novozhilov, K. F. Chernykh, and E. I. Mikhailovskii, The Linear Theory of Thin Shells (Politekhnika, Leningrad, 1991) [in Russian].

41. L. P. Lebedev, M. J. Cloud, and V. A. Eremeyev, Tensor Analysis with Applications in Mechanics (World Scientific, Singapore, 2010).

42. E. B. Wilson, Vector Analysis, Founded upon the Lectures of J. W. Gibbs (Yale Univ. Press, New Haven, 1901).

43. P. A. Zhilin, Vector and Second-Rank Tensors in Three-Dimensional Space (Nestor, St. Petersburg, 2001) [in Russian].

44. L. M. Zubov and M. I. Karyakin, Tensor Calculus. Foundations of the Theory (Vuzovskaya Kniga, Moscow, 2006) [in Russian].

45. E. I. Grigolyuk and I. T. Selezov, Nonclassical Theories of Rod, Plate, and Shell Vibrations in Results in Science and Technology. Mechanics of Deformable Solids, Vol. 5 (VINITI, Moscow, 1973) [in Russian].

46. E. I. Grigolyuk and P. P. Chulkov, Stability and Vibrations of Three-Layer Shells (Mashinostroenie, Moscow, 1973) [in Russian].

47. H. Altenbach, J. Altenbach, and W. Kissing, Mechanics of Composite Structural Elements (Springer, Berlin, 2004).

48. R. Szilard, Theories and Applications of Plate Analysis. Classical, Numerical, and Engineering Methods (Wiley, Hoboken, New Jersey, 2004).

49. C. M. Wang, J. N. Reddy, and K. H. Lee, Shear Deformable Beams and Shells (Elsevier, Amsterdam, 2000).

50. H. Altenbach and V. A. Eremeyev, "Eigen-Vibrations of Plates Made of Functionally Graded Material," CMC 9 (2), 153-178 (2009).

51. J. Chróścielewski, J. Makowski, and W. Pietraszkiewicz, Statics and Dynamics of Multifold Shells: Nonlinear Theory and Finite Element Method (Wydawnictwo IPPT PAN, Warsaw, 2004) [in Polish]. 
52. A. Libai and J. G. Simmonds, The Nonlinear Theory of Elastic Shells (Cambridge Univ. Press, Cambridge, 1998).

53. W. Pietraszkiewicz, Finite Rotations and Lagrangian Description in the Nonlinear Theory of Shells (Polish Sci. Publ., Warsaw-Poznan, 1979).

54. P. M. Naghdi, "The Theory of Shells and Plates," in Handbuch der Physik, Vol. 2, Ed. by S. Flügge (Berlin, 1972), pp. 425-640.

55. M. B. Rubin, Cosserat Theories: Shells, Rods, and Points (Kluwer, Dordrecht, 2000). 